\title{
XXIII.
}

\section{Ueber die cadaverösen Veränderungen der Altmann'schen Granula.}

\author{
(Aus dem Pathologischen Institut zu Berlin.)
}

Von Dr. Paul Dannehl in Berlin.

Die im Zellkörper schon seit längerer Zeit bekannten Eiweisskörner (Virchow) und Fäden (Kupffer, Flemming) sind dem. Interesse der mikroskopischen Forschung erheblich näher gerückt worden, seitdem durch die Ehrlich'schen Leukocytenfärbungen und die Altmann'schen Untersuchungen Methoden geboten waren, welche eine distincte Darstellung, in gewissem Maasse auch eine ungefähre chemische Charakterisirung dieser Elemente gestatteten. Während Ehrlich's Methoden die Färbung der Zellkörner - Granula - nur in Objecten zulassen, die im Deckglas-Trockenpräparat dargestellt werden können, setzte Altmann sich die erweiterte Aufgabe, dieselben auch in Organschnitten nachzuweisen, wodurch erst eine Untersuchung des ganzen Gebietes der Körner und Fäden in den Zellen möglich wurde.

Altmann's Methode ist bisher systematisch nur an .Objecten, die soeben dem lebenden Körper entnommen waren, verwandt worden, und dadurch ihre Anwendbarkeit in einer ihrer Bedeutung durchaus nicht entsprechenden Weise vornehmlich auf zoologische Objecte beschränkt worden. Altmann selbst schlägt vor, die zu fixirenden Stücke, um getreue Abbilder der während des Lebens vorhandenen Verhältnisse zu erhalten, möglichst dem frisch getödteten Thiere zu entnehmen. Das grosse Gebiet der Pathologie gestattet indessen eine solche Verwendung ganz frischer Objecte nicht, und es erklärt sich so, dass Granulauntersuchungen bei pathologischen Zuständen bisher kaum unternommen worden sind. Diese bisherige Lücke liess eine Untersuchung der cadaverösen Veränderungen der Granula und eine Bestimmung der Dauer des Ablaufs derselben sehr wünschenswerth erscheinen, abgesehen von dem rein wissenschaftlichen Interesse, das eine derartige Untersuchung hat. 
Wesentlich de erwähnte praktische Gesichtspunkt war es, der mich bestimmte, auf Anregung von Herrn Dr. 0. Israel das Verhalten der Granula im todten Körper näher za untersuchen. Es liessen sich von vornherein drei Möglichkeiten annehmen: 1. Die Granula erhalten sich im todten Körper längere Zeit and bleiben durch die Altmann'sche Methode fixirbar. 2. Sie bleiben im todten Körper vorhanden, lassen sich aber nicht mehr fixiren. 3. Sie versehwinden nach dem Tode darch ainen Auflösungsprozess. Zwischen den beiden letzterwähnten Möglichkeiten lässt sich eine Unterscheidung durch die mikroskopische Untersuchung kaum durchfibren, und es ist diese Frage anch von untergeordneter Bedeutung. Die Hauptfrage bleibt die, wie lange Zeit nach dem Tode überhaupt eine Fixirung und Färbung der Granula möglich ist.

Nach der von Al tmann angegebenen Methode - Fixirung mit Osmiumsäure und doppelt chromsaurem Kali, Färbung mit Säurefuchsin und Pikrinsäure - behandelte ich nun die verschiedenen Versuchsthieren entnommenen kleinen Organstücke, die ich verschieden lange nach dem Tode des betreffenden Thieres hatte liegen lassen. Um die zu erwartenden Veränderungen der Granula von etwaigen Fehlern bei der Färbung unterscheiden zu können, wurden mit Ausnahme der ersten Untersnohungsreihen (Maus, Triton) alle späteren Untersuchungen mit Zuhülfenahme von Controlfärbungen gemacht. Es wurden dazu Schnitte von Stücken genommen, die, wie schon festgestellt war, gut färbbare Granula enthielten. Ich lasse nun meine Untersuchungen folgen:

\section{Vorversuch.}

Zunächst wurden, um zu sehen, $o b$ die Veränderungen der Grauula sehr schnell nach dem Tode einträten, Stüoke von jungen Mäusen sofort, $\frac{1}{4}, \frac{1}{2}$, $\frac{3}{4}$ und 1 Stunde nach dem Tode fixirt und untersucht. Innerhalb dieser Zeit konnten Veränderungen der Granula nicht nachgewiesen werden.

Dies Resultat gestattete den Schluss, dass unmittelbar nach dem Tode die Granula sich nicht veränderten, eine Ansicht, die auch bereits von 0 . Israel ${ }^{1}$ ) ausgesprochen worden ist. Es

1) O. Israel, Die anämische Nelrose der Nieronepithelien. Dieses Arehị Bd. 123 . 
wurden die Untersuchungen demgemäss auf weitere Zeit ausgedehnt. Als Objecte dienten Organstiicke sowohl von Kalt- wie von Warmblütern, da es wichtig erschien, festzustellen, ob sich bei den ersteren, deren Lebensprozesse bekanntlich im Allgemeinen langsamer verlaufen, auch in Bezug auf das Verhalten der Granula Unterschiede herausstellen würden. Ich nehme der Uebersicht halber die Untersuchungen bei Kaltblütern vorweg, obgleich zwischen der ersten und zweiten Reihe derselben zeitlich Warmblüteruntersuchungen liegen.

\section{A. Kaltblüter.}

Triton.

Es wurden Organstücke von Tritonen sofort, 2, 4,6 und 8 Stunden nach dem Tode fixirt. Im Allgemeinen zeigen die urtersuchten Organe, Leber, Ovarium und kleinere Drüsen Granula von bedeutender Grösse, so dass meist schöne Bilder erhalten wurden. In der quergestreiften Körpermusculatur konnten bei der angewandten Vergrösserung Granula nur schwer gefunden werden, leichter in der. Herzmusculatur, wo dieselben beträchtlich grösser waren und sich in Reihen binter einander gelagert zwischen den Fibrillen gut verfolgen liessen. Veränderungen der Granula waren innerhalb der der Fixation voraufgegangenen Zeit noch nicht eingetreten.

\section{Salamander.}

Nach den noch zu erwähnenden inzwischen an Warmblütern gefundenen Resultaten setzte ich die Untersucbung an Kaltblütern fort, indem ich Leberstäcke von Salamandra maculata verschieden lange Zeit, bis zu $6 €$ Stunden, nach dem Tode bis zur Fixirung liegen liess. Etwas ungünstig gestaltete sich diese Untersuchung durch den grossen Pigmentreichthum der Salamanderleber. Das Pigment fand sich theils in feinsten Körnchen von der Grösse der Granula vertheilt oder in grösseren schwarzen Klumpen zusammengehäuft vor. Indessen wurde dieser Nachtheil durch die bedeutende Grösse der zelligen und granulären Elemente des Salamanders hinreichend ausgeglichen. Der grössere Theil des Raumes der Leberzellen wird von einem zusammenhängenden ausgedehnten Netzwerk eingenommen, dessen Bedeutung später bei der Katzenleber erörtert werden soll. Es entsteht durch das $\mathrm{Zu}-$ sammentreten von zablreichen schmalen, schwarzen Ringen, deren Centra hell und leer erscheinen. Zwischen den einzelnen Ringen, sowie in dem von ihnen nicht eingenommenen Theil der Zellen liegen dicht gedrängte, ziemlich grosse und scharf gefärbte Granula. Fast in jeder Zelle ist es deutlich sichtbar, wie die Granula in grösserer Menge die theils längs-, theils quergetroffenen Gallencapillaren umlagern, während der Kern inmitten des Netzwerkes liegt. Nach 20 Stunden sind alle Granula noch distinct gefärbt; ein Kälteversuch, der nach der gleichen Zeit so gemacht wurde, dass die betreffenden Stücke bis zu ibrer Fixation auf Eis gelegt wurden, 
ist deshalb aicht beweisend. Nach 30 Stunden erscheinen in fast allen Zellen distinct gefärbte Granula in baum rerminderter Anzahl. Auch nach 40 Stunden sind erbebliche Veränderungen der Granula nicht erkennbar. In der Peripherie versehtedener Zellen erscheinen dagegen eigenthiziche grau-schwarze Stellen, bald kleiner und rundlich, bald zu grösseren, unregelmässigg gestalteten Klumpen vereinigt. Von den erwähnten bellen Netzwerken in der Leber sind sie durch ibren grau-sehwaren lnkalt, ibre unregelonăssigere Form und ihre Lage in der Peripherie der Zellen wohl zu unterschoiden. Die erbaltenen Bilder sind dernach recht bunt, indem die grell rothen Granula, die tiefschwarzen Pigmenthaufen, die hellen Ringe und die grau-schwarzen Stellen in den meisten Zellen mit einander abwechsela. Nach 66 Stunden enthalten nur noch menige Zellen durchweg distinct gefärbte Granula, in den meisten ist nur noch eine undeutliche Körnung sichtbar. In diesen liegen hin uad wieder einzelne gröșere, schärfer gefärbte Granula. Die grau-schwarzen Stellen in der Peripherie der Zellen baben an Ausdehnung zugenommen, einzelne Zellen sind ganz davon erfült.

Eine bestimmte Deutung im Anschluss an die bekannten Beobachtungen von Kraus und Hauser, will ich diesen Erscheinungen nicht geben. Dass die grau-schwarzen Stellen in wen frischeren Stücken der Leber nicht vorhanden sind, ist gewiss. Doch wage jch hieraus Schlüsse nicht zu ziehen, da ja bei der Leber durch locale Fettinfiltrationen leicht Täuschungen hervorgerufen werden können. Es ist daher möglich, dass der ganze Befund ein zufälliger ist, indessea sei nochmals betont, dass ausschliesslich in dem zuletzt untersuchten Stück sich die beschriebenen Stellen vorfanden, was für die Annahme einer cadaverösen. Fettmetamorphose innerhalb der Zellen sprechen würde.

Es ist also nach 66 Stunden dio Grenze der Fixirbarkeit der Gravula bei Kaltblütern noch nicht erreicht worden, doch laben dieselben so gelitten, dass von ihrer ursprünglichen Anurdnung und Menge innerhalb der Zellen nichts mehr erkenubar ist. Bis zum Eintritt der gänzlichen Unfärbbarkeit derselben könnte indessen immerhin noch eine ziemliche Zeit vergehen.

Ich gehe nun zu den Untersuchungen an Warmblütern über.

\section{B. Warmblüter.}

Zur Untersuchang kamen Organtheile oiner Katze, eines Kaninchens, sowie menschliches Material.

\section{Ka t $z$ e.}

Es wurden Leber, Niere, Herz und Milz zu verschiedenen Zeiten, bis zu 20 Studen nach dem Tode fixirt. Es ergab sich dabei Folgendes: 


\section{Niere.}

An sofort nach dem Tode fixirten Stïcken sind sämmtliche Epitbelzellen der Harnkanälchen exfüllt mit dicht gedrängten, kleinen Granulis, die im Allgemeinen in radiären, von der Peripherie zum Lumen hinziehenden Reihen angeordnet sind. Die Granulareihen zieben an den Keruen rorbei, überragen sie aber nach dem Lumen hin nur um ein Geringes. In der Regel hört in der Zone jenseits der Kerne die regelmässige Anordnung der Granula auf, und es liegen nur noch vereinzelte, bald kleine, bald auch grössere Granula um die Kerne herum. In einzelnen Harnkanälchen finden sich auch in der Nähe des Lumens noch zablreiche Granula, bisweilen ist sogar das ganze Lumen damit erfüllt. Nach 8 Stunden sind die Contouren der Zellen und Kerne noch scharf, die Granula nocb überall deutlicb sichtbar. Indessen hat sich die regelmässige Anordnung an einigen Stellen schon verloren, und die Granula liegen zerstreut durch einander. Nacb 15 Stunden erscheinen die Körner bin und wieder verschwommen und geben eine mebr diffuse Färbung, meist sind sie aber noch distinct gefärbt. Auch nach 20 Stunden ist die Färbung meist noch gut gelungen. In einzelnen Harnkanälchen sind die Veränderungen weiter vorgeschritten: die Úmrisse der Zellen und Kerne haben sich verwischt, Granula sind nur noch durch eine diffuse Rothfärbung angedeutet. In anderen Zellen zeigt nur die Ungebung der Kerne noch dentliche, meist ziemlich grosse Granula, während der äbrige Zellraum nur eine undeutliche Körnung erkennen lässt. Die meisten Harnkanälchenepithelien aber sind noeh voll von Granulis; dieselben zeigen allerdings keine regelmässige Anordnung mebr, sondern liegen meist in dichten Haufen in der Peripherie der Harnkanälchen.

\section{Leber.}

Die Leberschnitte zeigen ähnliche Verhältnisse. Die Zellen enthalten ziemlich grosse, im Verbältniss zu den Epithelien der Niere allerdings nur spärliche Granula, die fast überall eine regelmässige Anordnung anfweisen: In einfacher ringfömiger Lage finden sie sich in der unmittelbaren Umgebung der Kerne, in stärkerer Anbäufung liegen sie um die Gallencapillaren und ziehen in einzelnen Strängen durch den sonst von Körnern freien Zellkörper bindurch. Nach 8 Stunden ist in der Menge, sowie der Anordnung der vorbandenen Granula noch keine Veränderung wahrzunebmen, erst bei einer 15 Stunden nach dem Tode erfolgten Fixation erscheint die Anordnung der Zellkörner nicht mehr so regelmässig wie vorher. Dieselben liegen mehr in einzelnen Anhäufungen in der Peripberie der Zellen zusammen und heben sich schon bei schwacher Vergrösserung als dunkle rothe Flecke von den belleren, körnchenfreien Stellen ab. Nach 20 Stunden hat sich das Bild noch nicht sebr verändert, die Anzahl der Granula bat sich kaum vermindert. Einzelne wenige Zellen lassen deutliche Granula nicht mehr erkennen, sondern enthalten nur schwach körnige, braungelbe Massen.

In den Leberschnitten finden sich im Innern der Zellen die schon beim Salamander erwähnten hellen kreisförmigen, wie 
Vacuolen ausisehenden Stellen, die gewöhnlich von einem schmalen schwarzen Rand umsäumt sind und zu bald mehr, bald weniger' ausgedehnten Netzwerken zusammentreten, während die Granula auf den übrig bleibenden Theil der Zelle, sowie die $Z$ wischenräume zwischen den Maschen des Netzwerkes beschränkt sind. In der Lober nehmen die hellen Ringe meist dio mittlere Zone der Zellen, gewöhnlich also die Gegend um die Kerne ein. Dass sich diese scheinbaren Vacuolen erst nach dem Tode bilden oder wenigstens vermehren, wie es mir nach der Betrachtung der ersten Schnitfe der Fall zu sein schien, hat sich bei den weiteren Untersuchungen nicht bestätigt. Es hätte sich ảiese Annahme eines cadaverösen Vorganges nur durch ein post mortem stattindendes Fortlaufen des functionellen Prozesses in der Zelle oder eine cadaveröse Metamorphose des Zellinhaltes begründen lassen. Das Erste erschien an und für sich zu unwahrscheinlich, und auch das Zweite musste verworfen werden, da sich auch in unmittelbar nach dem Tode fixirten Stücken solche hellen Ringe, mitunter sogar, wie beim Salamander, in grosser Ausdehnung vorfanden. Auch die Extraction der in den Lücken vorhanden gewesenen Inhaltsmassen blieb bei der Annahme eines cadaverösen Vorganges unerklärt. Es blieb daher wur der Schluss übrig, dass die gefundenen Bilder den Verhältnissen im Moment des Absterbens des Versuchsthieres entsprachen. Die in verschiedenen Stücken desselben Organes vorhandeven Unterschiede in Bezug auf die Anzahl und Grösse der hellen Lücken lassen sich dann auf Verschiedenheiten, die schon im Leben innerhalb der einzelnen Zellengebiete vorhanden waren, zurückfuhrea. Altmana selbst boschreibt solche Netzwerke und erklärt ihre Entstehung folgendermaassen: die ursprünglichen Granula wandeln sich durch Fettassimilation in grössere Kugeln um, die sich mit Osmiumsäure schwärzen. Diese schwärzende Eigenschaft kommt der Osmiumsäure aber nicht allen Fetter, sondern nur dem Oleïn und der Oelsäure gegenüber za. Neben dem Rest der ursprünglichen Gränulasubstanz können also nur diese beiden in den geschwärzten Kugeln vorhanden sein. Die Lösung und Extraction des im Centrum der Kugeln vorhandenen

i) R. Aitmann, Die Elementarorganismen und ihre Beziehungen zu den Zellen. Leiprig 1890. 
Inhaltes geschieht durch längere Einwirkung des Alkohols, wonach die schwarzen leeren Ringe zurückbleiben. Diese Löslichkeit in Alkohol nach der Osmiumbehandlung hat das Oleïn nicht, wohl aber die Oelsäure. Danach hat die Oelsäure den central gelegenen Inhalt der nach der Alkoboleinwirkung leer zurückbleibenden schwarzen Ringe gebildet, die ihrerseits aus Oleïn bestehen müssen. - Diese Erklärung, glaube ich, trifft auch für meine Bilder zu, und es ist die Extraction der ursprünglichen Inhaltsmassen der Ringe auf den Alkohol zurückzuführen.

Im Allgemeinen hatten also die Granula nach Ablauf von 20 Stunden nur wenig gelitten, jedenfalls die Grenze ibrer Fixirbarkeit noch bei weitem nicht erreicht. Es lag daher Grund vor, anzunehmen, dass die Veränderungen der Granula mit der Verlängerung der Frist nach dem Tode des Thieres bis zu ihrer Fixirung in derselben langsamen Weise weiter fortschreiten wärden. Bis zum Eintritt der vollständigen Unfärbbarkeit der Granula konnte demnach noch eine geraume Zeit vergehen, und es wurde daher bei dem nächsten Versuchsobject, einem Kaninchen, die bis zur Fixation der gewählten Stücke verstreichende Frist erheblich erweitert.

\section{Kaninchen.}

Es wurden Leber und Niere eines Kaninchens sofort, 24 und 48 Stunden nach dem Tode fixirt.

I eber.

In Scbnitten von der Leber sind bei sofort nach dem Tode vorgenommener Fixirung alle Zellen voll von Granulis, doch fallt hier ein erbeblicher, in den vorher untersuchten Organen nicht bemerkter Grössenunterschied der Granula in den verschiedenen Zellen auf: die meisten enthalten kleine und dicht gedrängte Granula, $z$ wischen denen hier und da einzelne grössere erscheinen, einzelne $Z$ ellen dagegen zeigen durchweg beträchtlich grössere und viel weniger dicht liegende Körner. Alle Granula sind gleichmässig in den Zellen vertheilt. Die 24 Stunden nach dem Tode erhaltenen Bilder zeigen schon bemerkenswerthe Verändertugen. In fast alleu Zellen baben sich noch Granula gefärbt, aber sie sind vielfach blasser und grenzen sich nicht mehr so scharf gegen das intergranuläre Gewebe ab. Schärfer und intensiver sind die grösseren Granula gefärbt, die zwischen den kleinen in grösserer oder geringerer Anzahl bervortreten. Nur wenige Zellen, ungefähr 5 bis 5 in jedem Gesichtsfeld, entbalten durchweg gut gefärbte, grosse Granula und heben sich durch ihre intensive Färbung auf den ersten Blick von ihrer 
Umgebung $a b$. In wepigen Zellen endlich, in dener auch die Kerne schon gelitten baben, ist eine distincte Färbung der Granula nicht mebr eingetreten. In den 48 Stunden nach dem Tode fixirten Stäcken sind Kern und Rernkörperchen in den meisten Zellen nicht mehr scharf begrenzt. Granula sind nur noch an einzelnen Stellen in geringer Anzabl vorhanden, sie sind Llass und undeutlich. In den meisten Zellen sind sie überbaupt nicht mehr sichtbar, wenn auch eine Differenzirung des Zellkörpors gegenüber den Kern insofern eingetreten ist, dass der erstere dunkler gefarbt erscheint und eine undeutliche Körnung erkennen lässt.

\section{Niere.}

In der Niere sind die Granula unmittelbar nach dem Tode ziemlich regelmässig angeordnet. Sie liegen in radiär verlaufenden Reihen und zieben in gerader Richtung an den Kernen vorbei. In einzelnen Harnkanälchen überwiegen grössere Granula, die dieser Anordnung nicht folgen, sondern ringförmig um die Kerne liegen. Ein bestimmtes Verbalten der Granula zu dem Kern lässt sich hier, wie anch an anderen Nieren- sowie Leberschnitten, nicht nachweisen. Oft findet sich um den Kern eine ganz schmale Zone, die frei von Granulis erscheint und von einem Granularing umgeben wird, oft aber, namentlich in der Leber, lagern sich die Granula unmitelbar an den Kern heran. In den vorliegenden Schnitten liegt in der Regel zwiscben Kern und Granulis eine schmale Zone körnchenfreier Substanz. Ausser in den Harnkanälchen sind auch in den Glomerulis in einzelnen Epithelzellen Granula deutlich sicbtbar. Die nach 24 Stunden eingetretenen Veränderungen entsprechen denen in der Katzenniere. Granula sind noch in allen Zellen vorhanden, doch fehlt meist ibre regelmässige Anordnung, sie liegen vielmehr in Klumpen zusammen an der Basis der Epithelzellen. Am besten sichtbar sind die grossen, zerstreuten Granula, wie noch an vielen Stellen als isolirte, deutlich ringförmige Lage um die Kerne hervortreten und auch innerhalb der Lumina angetroffen werden. Nach 48 Stunden sind deutliche Granula nicht mebr sichtbar. An einzelnen Stellen erkennt man noch diffuse Rothfärbungen, an anderen hat eine gleichmässige Gelbfärbung des ganzen Gewebes stattgefunden.

Es kann also auch die Frist von 48 Stunden vom Tode des Thieres an bis zur Vornahme der Fixirung noch nicht als die ausserste Grenze der Fixirbarkeit der Granula angesehen werden, wenngleich nach Ablauf dieser Zeit nur noch ein schwacher Rest des ursprünglichen Körnerinhaltes der Zellen vorhanden ist. Jedenfalls sind gute Granulabilder nach 48 Stunden bei warmblätigen Thieren nicht mehr zu erwarten.

\section{Menscb.}

Es lag wahe, die für die Warmblüter bisher gefundenen Ergebnisse ohe weiteres auf den Menschen zu übertragen. In- 
dessen zeigen die Verhältnisse bei diesem doch soviel Abweichungen von dem Typus der übrigen Warmblüter, dass es gerathen erschien, auch mit menschlichem Material eine besondere Untersuchung anzustellen. $\mathrm{Zu}$ diesem Zwecke wurden Leber und Niere von vier verschieden frischen Leichen fixirt und mit einander verglichen. Die Resultate waren zunächst wenig befriedigend. Bei Schnittdicken, die bei den vorhergehenden Versuchen durchaus gelungene Bilder gegeben hatten, erschien in zahlreichen Schnitten der Zelleninhalt nur undeutlich und diffus gefärbt. Da die mitgefärbten Controlpräparate etwaige Fehler der Färbung auszuschliessen gestatteten, wurden die Versuche mit möglichst dünn hergestellten Schnitten - etwa $1 \mu$ - wiederholt. Es ergab sich dabei Folgendes:

Erste Leiche, 10 Stunden alt.

Niere: In der Niere sind in allen Zellen Granula vorbanden, aucb tritt nur die Minderzahl derselben mit wünschenswerther Klarheit aus ibrer Umgebung hervor. Diese scharf gefärbten Granula liegen gewöbnlich in der Umgebung der Kerne.

Leber: Die Bilder von der Leber sind besser, sie zeigen in fast allen Zellen distinct gefärbte, dicht liegende Granula. Meist sind dieselben kleiner als die bisher in den Leberzellen beobachteten Körnchen.

Von derselben Leiche wurden entsprecbende Stücke auch 24, sowie 75 Stunden nach dem Tode fixirt:

Nach 24 Stunden hat sich die Anzahl der Granula in den Nierenzellen kaum vermindert, doch liegen dieselben mehr in einzelnen Anhäufungen in der Peripherie der Harnkanälchen; während diese sonst nur vereinzelte Granula enthalten. In der Leber hat sich das Bild kaum verschlecbtert.

Bei der 75 Stunden nach dem Tode vorgenommenen Fixirung, sind in den Nierenzellen Granula, mit Ausnabme einiger rother Punkte, die sich aus dem gleichmässig gefärbten Zelleninhalt abheben, nicht zu sehen. In der Leber zeigen alle Zellen noch eine undeutliche Körnung, der Zelleninhalt ist auch hier gleichmässig gefärbt. Die schon beim Salamander erwähnten, in der Peripherie der Zellen auftretenden, rundlichen grau-schwarzen Stellen, die bald isolixt, baid zu unregehnässig gestalteten Klumpen vereinigt liegen, finden sich auch hier. Einzelne Zellen sind ganz von ihnen eingenommen. In den Nierenschnitten sind sie nicht vorhanden.

\section{Zweite Leiche, 12 Stunden alt.}

$\mathrm{Niere:} \mathrm{In} \mathrm{allen} \mathrm{Harnkanälchen} \mathrm{finden} \mathrm{sich} \mathrm{Granula} \mathrm{in} \mathrm{ziemlicher} \mathrm{An-}$ zahl, doch unregelmässig vertheilt und ausnabmslos so klein, wie sie bisber, ausgenommen in quergestreiften Muskeln, von mir noch nicht beobachtet wurden. Es gelang daber nur an ansgesncht dünnen Schnitten und bei 
starker Differenzirung, sie mit der nöthigen Karheit darzustellen. Zwischen ihnen liegen zahlreiche grössere und kleinere schwarte Kugelt.

Leber: Anch in dep Leber sind Granula wegen ihrer ansserordentichen Kleinheit nor scbwer darzustellen. Doch finder sie sich an geeigneten Schnitten in fast allen Zellen vor, meist allerdings nur in spärlicher Anzahl. Vereinzelt finden sick auch grössere Granula.

\section{Dritie Leiche, 36 Stunden alt.}

Nicre: In der Niere haben sich überall Granula von mittlerer Grösse distinct gefarbot. In grösserer Anzabl liegen sie una in der Umgebung der Berne, sonst sind sie nur vereinzelt sichtoar.

Le ber: In der Leber sind deutliche Granula nur in sehr geringer Anzahl vorhanden, meist sind die Zellen mit zahlreichen grossen und kleinen schwarzen Kugeln erfüllt.

\section{Vierte Leiche, 52 Stunden alt.}

Niere: In allen Zellen sind noch Granula sichtbar. In einigen ist die Färbung mehr diffus, in den meisten aber heben sicb die Körner scharf von der Grundsubstanz ab.

Leber: Auch die Leberschnitte zeigen in fast allen Zelled noch Granula. Dieselben sind in Allgemeinen recht klein und verschwimmen desbalb etwas, doch finden sich zwischen ihnen auch grössere, scharf gefärbte Körner in erheblicher Anzahl. Nur in wenigen Zellen sind Granula nicht mehr zu erkenuen.

Im Allgemeinen stimmen die Resultate beim Menschen mit den bei den vorhergehenden Warmblüteruntersuchungen erhaltenen überein, es war sogar in den 52 Stunden nach dem Tode fixirten menschlichen Theilen die Granulafärbung erheblich besser ausgefallen als in den 48 Stunden nach dem Tode fixirten Kaninchentheilen, ein Punkt, auf den ich noch zurückkommen werde. Ob die auffallenden Grössenunterschiede der Granula in den verschiedenen Stäcken, namentlich ibre Kleinheit in dem der $\%$ weiten Leiche entnommenen Material, zufällige sind oder eine innere Ursache haben, will ich nicht entscheiden. Aber die überraschende Winzigkeit der Granula, sowie der Umstand, dass die betreffende Person an einem septischen Prozess zu Grunde gegangen war, lassen sich vielleicht in Beziehung zu einander bringen, und es dürften weitere Untersuchungen über den Einfluss bestimmter Gifte auf die Granula noch Licht in diese Frage bringen.

Ein erheblicher Unterschied zwischen Kalt- and Warmblütern in Bezug auf die Zeit, innerhalb welcher die Granula sich 
nicht mehr färben, hat sich durch die Untersuchung nicht feststellen lassen. Indessen sind die beim Salamander 66 Stunden nach dem Tode erhaltenen Bilder, was die Färbung der Granula anbetrifft, mit den 48 Stunden alten vom Kaninchen noch mindestens auf eine Stufe zu stellen, und man wird also berechtigt sein, auf ein etwas langsameres Ablaufen der Granulaveränderungen bei Kaltblütern zu sehliessen.

Fasse ich die Ergebnisse meiner Arbeit zusammen, so lässt sich über das Verhalten der Granula im todten Körper Folgendes sagen: Das Auftreten der ersten Leichenveränderungen an den Granulis giebt sich kund durch das Verschwinden der im Leben vorhanden gewesenen charakteristischen Anordnung derselben. Sie rücken dichter aneinander, lassen sich durch die Färbung nicht mehr so scharf darstellen und färben sich immer diffuser. Bis zum Auftreten dieser ersten Veränderungen vergeht eine Zeit von 18-20 Stunden, bis zum Abschluss derselben, d. h. bis zur völligen Unfärbbarkeit der Körnchen eine solche von 50-60 Stunden.

Der Eintritt und das Fortschreiten der Leichenveränderungen der Granula geschehen nicht in allen Zellen gleichmässig, sondern einzelne Zellen, oft auch einzelne Zellengebiete, zeigen stärkere, andere geringere Grade der Veränderung. In den ersteren kommt es bald zu einern vollständigen Verschwinden der Granula in den Zellen, es bleibt eine gleichmässige braungelbe Färbung der Zelle, in der mitunter noch eine schwache Körnung erkennbar ist, zurück. An andern Stellen erhalten sich die Zellkörner länger, namentlich erscheinen einzelne grössere Granula oft noch allein deutlich gefärbt. Diese überragen nicht selten durch ihre Grösse die vorher vorhanden gewesenen Granula um ein Bedeutendes. Zur Erklärung dieser Erscheinung möchte ich auf eine Hypothese Israel's in seiner schon wiederholt erwähnten Arbeit hinweisen. Er beobachtete in der Kaninchenniere ähnliche grosse Körner und kam za der Ausicht, es möchten die in der Grundsubstanz eingeschlossenen Granula flüssigen Aggregatzustand besitzen, ähnlich dem Fett und dem Nervenmark, und ihre Form nur durch die zähere Grundsubstanz erhalten. Wird letztere nun weicher, widerstandsunfähiger, wie es wohl mit der Zunahme der nach rem Tode des Thieres bis 
zur Fixirung verflessenden Zeit der Fall sein wird, so können die flüssigen Körner austreten, confluiren und bilden so die grösseren isolirten Föruer.

Einen Zusammenhang zwischen den Veränderungen an den Granulis und denen an den Kernen habe ich nicht immer beobachten können. Indessen glaube ich einen solchen doch annehmen za dürfen. Hammer ${ }^{1}$ ) in seinen Untersuchungen über die nach dem Tode am Kern auftretenden Veränderungen kommt zu dem Schluss, dass bis zum völligen Verschwinden der Kerntheilungsfiguren in der menschlichen Leiche mindestens 48 Stunden vergehen. Diese Frist trifft für das völlige Unfärbbarwerden der Granula nicht genau zu, die Grenzen der. Färbbarkeit sind für dieselben etwas weiter hinaus, bei 60 Stunden gelegen. Doch entspricht dieses Verhältniss völlig den Befunden Israel's in nekrotisch sich umwandelndem Material, das auch eher Veränderungen der Kerne als solche der Granula aufweist. Man kann also wohl annehmen, dass im todten Körper die Veränderungen an dem Zellkern und im Zellenleib einander proportional verlaufen, nur ist das Tempo der Veränderungen am Kern etwas beschleunigter. Zum Schluss erfülle ich die angenehme Pflicht, Herrn Dr. O. Israel, erstem anatomischen Assistenten am pathologischen Institut zu Berlin, für die Anregung zn dieser Arbeit und die freundliche Unterstütaung bei der Anfertigung derselben meinen besten Dank auszusprechen ${ }^{2}$ ).

1) Ham mer, Ueber das Verhalten der Kerntheilungsfiguren in der menschlichen Leiche. Inaug.-Dissert. Berlin 1891.

2) Während der Abfassung dieser Arbeit kam wir eine Notiz eines italieniseben Autors, Zoja, Intorno ai Plastiduli fuscinofili, Leipzig 1890, zu Gesicht. Danach hat derselbe 24 Stunden nach dem Tode weder in der Anordnung noch in der Färbbarkeit der Granula eine Veränderung gefunden und nocb 60 Stunden nach dem Tode Granulafärbungen erhalteu. Meine Resultate sprechen im Allgemeinen dagegen: Nach 24 Stunden babe ich meist die ersten Veränderungen dor Granula schon eintreten sehen, und eine distincte Färbung derselben nach 60 Stunden ist mir nur bei Kaltblütern in vereinzelten Zellen gelungen, ich habe also im Ganzen etwas engere Grenzen für die Färbbarkeit der Granula gefunden. 